\title{
Trash Separator
}

\section{Shivam Agarwal, Shrishti Agarwal}

\begin{abstract}
According to statistics, India generates 62 million tons of waste (mixed waste containing both recyclable and non-recyclable waste) every year. Therefore, the proper disposing system is a crucial factor in a green and smart city. Implementing the recycling process efficiently is one of the major aspects that require attention, With the latest technologies, this garbage sorter bot is able to differentiate between different types of trash like plastic, metal, glass, etc. and traverse through its pre-defined path to drop it at its destination. FirebirdV, used for garbage separation is an autonomous bot and it is 5th among its series. Due to its versatility, it is widely used. It has ATMEGA 2560 (AVR), P8V5IRD2(8051), and LPC2148 microcontroller. The software part includes OpenCV, Deep Learning and other similar technologies. In programming, AVR studio (having open $\mathrm{C}$ compiler) is used for generating the hex file which is then loaded onto the robot. Then the predicted lists are served to the bot through serial communication. Finally, the bot puts the garbage in the desired waste collection basket.
\end{abstract}

Index Terms - Deep Learning, Firebird V (ATMEGA2560), OpenCV (Image Processing), Transfer Learning.

\section{INTRODUCTION}

This bot can be used in garbage houses, large dumping area, or any open place where garbage is scattered.

* Firstly, an image of the area is given to the program which extracts the image of garbage and dustbin from the image and their respective locations and predicts the garbage type and its corresponding dustbin type present. Then it passes their location to the bot.

* The bot uses the greedy or shortest distance approach to pick the garbage and place it in the nearest respective dustbin.

* Now, all the garbage is cleared and placed in the respective dustbin for correct disposal. The whole process does not require any type of labor or supervision.

Shivam Agarwal, ECE, KIET Group of Institutions, Ghaziabad, India, +91-7379445111

Shrishti Agarwal, ECE, KIET Group of Institutions, Ghaziabad, India, $+91-7289069616$

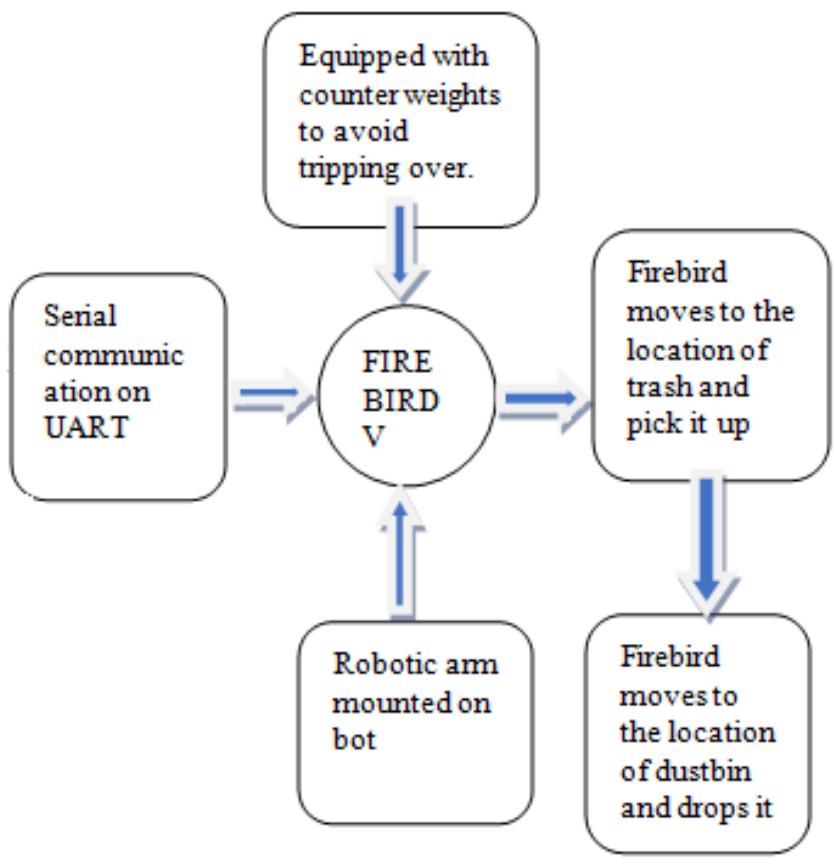

Fig 1: Block Diagram

\section{SOFTWARE}

Programming section can be been divided into three major categories i.e. Image processing, Deep Learning, and robotics. The code was written in two languages python (OpenCV, Pytorch) and Embedded C.

\section{A. Image Processing}

OpenCV is a library that consists of functions mainly aimed at computer vision is used here for pre-processing (morphological transformation, Gaussian Blur, etc.) .Firstly, the image of the area obtained is resized to a set dimension then the images of trash and dustbins are extracted termed as contours. These cropped images are then resized in the original size and then fed into the model for prediction. Image, contours, hierarchy $=\mathrm{cv}$ 2. findContours (thresh,cv2 .RETR_TREE, cv2.CHAIN_APPROX_SIMPL)

\section{B. Deep Learning}

Dataset, we used had more than 10000 images divided into various categories like glass, plastic, metal, etc. Dataset is divided into two sets i.e. train set (having $80 \%$ of total images) and test set (consisting of remaining 20\%). But before dividing it into above sets the whole dataset should be shuffled to and various transformations should be applied like random horizontal flip, random resize crop then convert it into tensor and then normalize it to improve the model accuracy and also increase the length of the dataset to make it less prone to overfitting.

The following figures are some images from our dataset. 


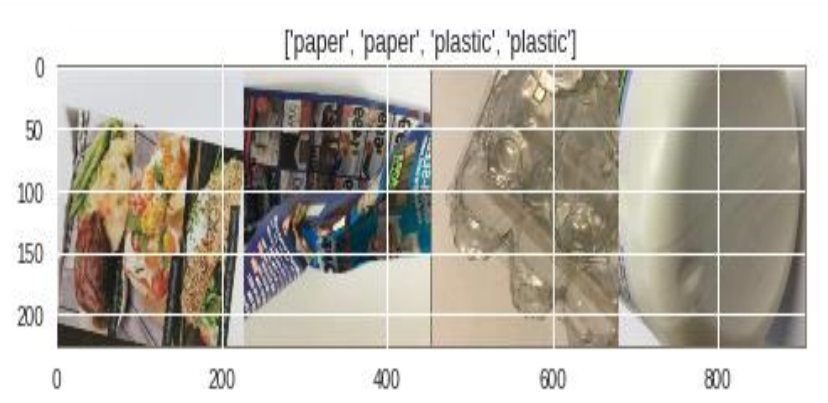

Fig 2 : Dataset

As transfer learning is the reuse of pretrained model used for a similar task that we are going to use it for, and it enables us to train a deep neural network with comparatively much less data. Also, it saves a lot of time and gives higher accuracy than training a network from scratch and uses lesser computational power to train.

For recognition, pretrained model ResNet (Residual Network) which was trained on ImageNet - 1000 dataset which has been used for training and trash classification is a similar task as performed by ResNet that is to classify different objects thus we have used it. An experiment shows that ResNet model, especially ResNet-101 (have 101 layers), here we have used ResNet as feature extractor by freezing the weights of all the layers except the final fully connected layer and replaced the weights of final fully connected layer by random weights and only this layer is trained, produces remarkable results i.e. having an accuracy of $93.33 \%$.
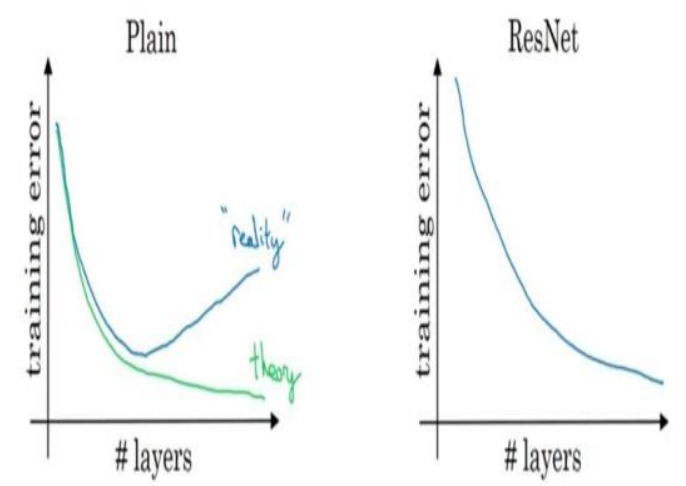

Fig 3: Training error graph

The above figure demonstrates that in ResNet, training deep layers decreases the training error in the last layers due to skip connection. Skip connection is a property which feeds the output of earlier layers to the deeper layers so that the properties of original content is not lost and which helps to classify minute details easily. We have used Stochastic Gradient Descent (SGD Optimizer) because it does the calculation at very high speed and updates the parameters frequently.

Hyperparameter was tuned until we have found the most satisfactory result. The model is then saved for future predictions. For testing, we have predicted many pictures and we found an amazing result and almost all the trash was classified correctly.

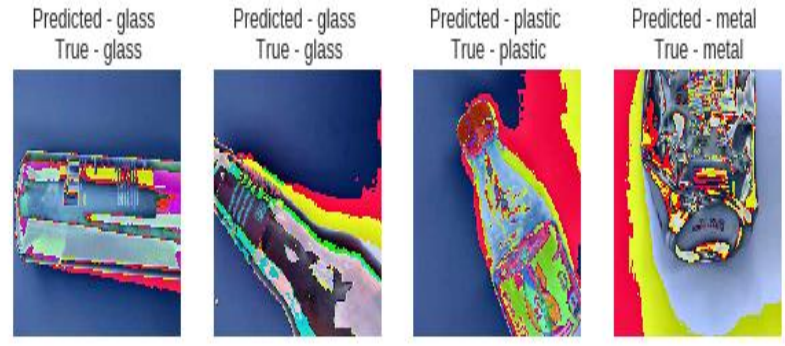

Fig 4: Prediction on test set

C. Robotics

The language is used to program the robot is Embedded C. In this we have to give values of different registers to get the required task done by the robot like

1. EIMSK = EIMSK $\mid 0 \times 20$; to enable Interrupt INT5 for right position encoder

2. ADCSRA = ADCSRA $\mid 0 x 40$; to set start conversion bit

3. ADCSRA $=$ ADCSRA $\mid 0 \times 10$; to clear ADIF (ADC Interrupt Flag) by writing 1 to it

further, we use these and more registers to perform the desired operation. We have programmed white line sensors and servo motors to make over bot perform the task we have found the nearest coordinate of trash then programmed to pick it up and place it in the nearest respective dustbin.

\section{Design Methodology}

In this project, ATMEGA 2560 based FIREBIRD V is used as an autonomous bot. It is coded in Embedded $\mathrm{C}$ which forms the brain of the bot. It is used for traversing the area from the source to the destination. [1]The bot uses the White Line Sensor to follow the path. In day time white line sensor should be surrounded by the black paper for its proper working.

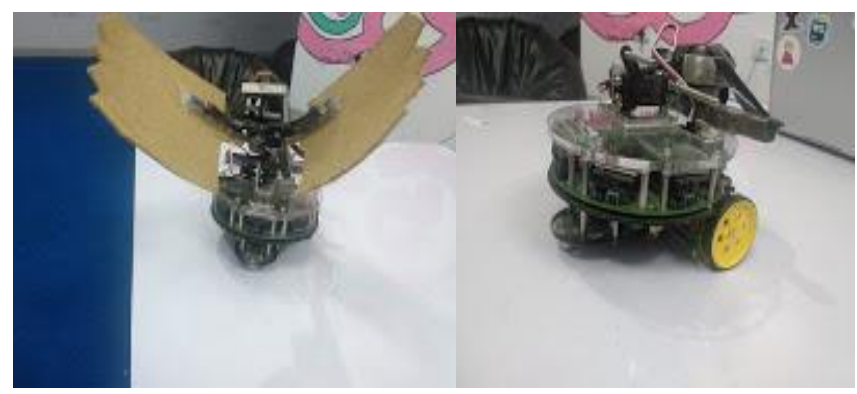

Fig 5: Robot

For the movement of the robot we have seen that to move forward, both the wheels should be in the same forward velocity. To move backward both the wheels should have the same backward velocity. To take soft right the right wheel should be at a slightly slower speed than the left wheel. To take hard right, the right wheel should rotate backward as the same speed left wheel is moving forward and vice versa for soft left and hard left.

We have designed an arm mechanism that is mounted at the center of the bot so that it could be aligned perfectly with the trash. The weight of the arm is strictly managed to avoid any mechanical failure. The bot is also equipped with some counterweights at its hind end to avoid tripping over. The arm performs three functions i.e. picking, holding and dropping. The robotic arm has two servo motors. the first one is 
International Journal of Engineering and Technical Research (IJETR)

ISSN: 2321-0869 (O) 2454-4698 (P) Volume-9, Issue-5, May 2019

mounted on the bot which helps in moving the whole arm vertically. The other one is mounted as an end factor. It opens and closes its claws for picking and dropping the trash. The rough design of the mechanism is shown in the figure.
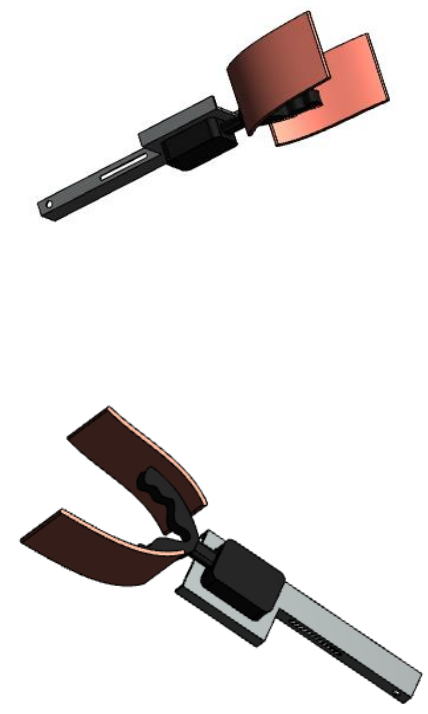

Figure 6: 3D image of open arms
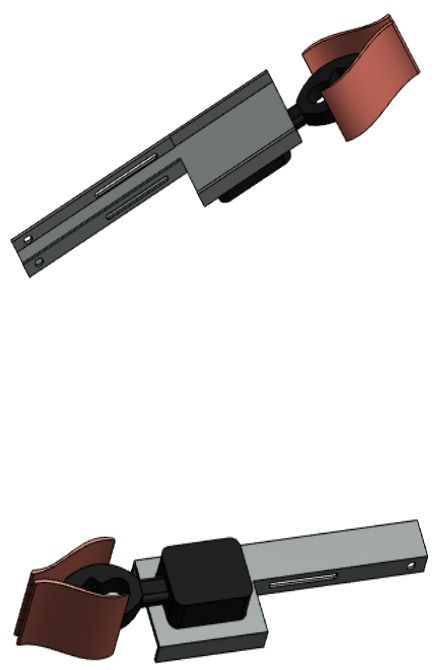

Figure 7: 3D image of closed arm

Other than these, Castor wheel is provided to give support to the bot for smooth movement.

\section{RESUlTS}

We have trained our model on different types of pretrained CNN architecture like ResNet-101, ResNet-50, ResNet-18, ResNet-152.

Different accuracies of these models are:

\begin{tabular}{|l|l|}
\hline \multicolumn{1}{|c|}{ Model } & \multicolumn{1}{|c|}{ Accuracy } \\
\hline ResNet101 & $93.93 \%$ \\
\hline ResNet152 & $91.9 \%$ \\
\hline ResNet18 & $88.7 \%$ \\
\hline ResNet50 & $89.56 \%$ \\
\hline
\end{tabular}

Table 1: Accuracy on different resnet models

For better tuning we have trained the above models using different values of hyperparameters.

\begin{tabular}{|l|l|}
\hline Hyper parameters & Best value \\
\hline Step size & 5 \\
\hline gamma & 0.1 \\
\hline Learning rate & 0.001 \\
\hline momentum & 0.9 \\
\hline
\end{tabular}

Table 2: Hyperparameters for best accuracy found

Due to the castor wheel we got a smooth movement, and due to big mouth of grabbing mechanism we were nearly able to hold and pick up all the trash one by one.

\section{CONCLUSION}

The bot could potentially prove to be a vast asset in the future, especially for a country like India, where the garbage produced is great both in quantity and variety. If commercialized, the bot could make the mammoth task of waste segregation a real and achievable target.

Of course, the bot cannot do so in its current state; a number of upgrades will be required and are being worked upon. Currently, its accuracy for recognizing different types of garbage is just a mere $93.98 \%$, but we feel the model works as a great prototype for showcasing its potential as an asset to the country.

This bot, which is the culmination if immense hard work from all the members, present an innovative and effective method to deal with garbage, which threaten the planet as a whole.

\section{REFERENCE}

[1] RM.Nachammai , N.Mrujool Kansara ,G.Lavanya ,R.Gopalakrishnan "White Line Follower Using FireBird V Robot" IJSRD-Internationa Journal for Scientific Research and Development Vol. 3, Issue 10, 2015 